\title{
Oxidative stress and mitochondrial damage: importance in non-SOD1 ALS
}

\author{
Maria Teresa Carri ${ }^{1,2}{ }^{*}$, Cristiana Valle ${ }^{2,3}$, Francesca Bozzo $^{1,2}$ and Mauro Cozzolino ${ }^{*}$ \\ ' Department of Biology, Università di Roma Tor Vergata, Rome, Italy \\ ${ }^{2}$ Fondazione Santa Lucia, IRCCS, Rome, Italy \\ ${ }^{3}$ Institute of Cell Biology and Neurobiology, IBCN, National Research Council, CNR, Rome, Italy \\ ${ }^{4}$ Institute of Translational Pharmacology, National Research Council, CNR, Molecular Mechanisms of Neurodegenerative Diseases, Rome, Italy
}

\section{Edited by:}

Manoj Kumar Jaiswal, Center for

Neuroscience and Regenerative

Medicine, USA

Reviewed by:

Rasna Sabharwal, University of

lowa, USA

Ellen F. Barrett, University of Miami

Miller School of Medicine, USA

Adrian Israelson, Ben-Gurion

University of the Negev, Israel

\section{*Correspondence:}

Maria Teresa Carri, Department of

Biology, Università di Roma Tor

Vergata, Via della Ricerca

Scientifica 1, 00133, Rome, Italy

e-mail: carri@bio.uniroma2.it;

Mauro Cozzolino, Institute of

Translational Pharmacology,

National Research Council, CNR,

Molecular Mechanisms of

Neurodegenerative Diseases, Via

del Fosso del Cavaliere 100, 00133,

Rome, Italy

e-mail: mauro.cozzolino@ift.cnr.it
It is well known that mitochondrial damage (MD) is both the major contributor to oxidative stress (OS) (the condition arising from unbalance between production and removal of reactive oxygen species) and one of the major consequences of OS, because of the high dependance of mitochondrial function on redox-sensitive targets such as intact membranes. Conditions in which neuronal cells are not able to cope with MD and OS seem to lead or contribute to several neurodegenerative diseases including Amyotrophic Lateral Sclerosis (ALS), at least in the most studied superoxide dismutase 1 (SOD1)-linked genetic variant. As summarized in this review, new evidence indicates that MD and OS play a role also in non-SOD1 ALS and thus they may represent a target for therapy despite previous failures in clinical trials.

Keywords: Amyotrophic Lateral Sclerosis, ALS, mitochondria, oxidative stress, neurodegeneration

\section{INTRODUCTION}

Amyotrophic Lateral Sclerosis (ALS) is a fatal motor neuron disease occurring less than once in 10000 people and thus classified as a rare disease. ALS is not purely a motor neuron disease and neuroinflammation and muscle degeneration also contribute to the non-cell autonomous death of motor neurons. As for other neurodegenerative diseases, ALS is sporadic in most cases, with a clear genetic origin in about $10 \%$ of patients, and is clinically heterogeneous in age and site of disease onset as well as rate of disease progression.

Different mechanisms such as protein aggregation, oxidative stress (OS), mitochondrial damage (MD), excitotoxicity and RNA dysmetabolism seem to contribute to ALS. Evidence on the relevance of these mechanisms comes in large part from models based on the expression of ALS-linked mutant superoxide dismutase 1 (SOD1). However, mutant SOD1 causes less than $1 \%$ of ALS cases and the list of mutant proteins found in ALS patients has grown noticeably in the last decade (Marangi and Traynor, 2014), to include a number of proteins related to cell functions as diverse as RNA metabolism (TDP-43, FUS/TLS, Senataxin,

Abbreviations: ER, endoplasmic reticulum; ROS, reactive oxygen species; RNS, reactive nitrogen species; PDI, protein disulphide isomerase.
Ataxin2, HNRNPA2/B1, ELP3, HNRNPA1), vesicle trafficking (Alsin, FIG4, OPTN, VABP, CHMP2B) and proteasomal function (UBQLN2, VCP). Noticeably, almost half of the familial cases are linked to a non-coding sequence, i.e., to an expanded hexanucleotide repeat in the C9orf72 gene.

Because mutations in SOD1, an ubiquitous superoxide scavenging enzyme, were the first identified cause of familial ALS in 1993, OS has been an obvious candidate to explain the pathogenesis of this disease. MD was also demonstrated in patients and in mutant SOD1 models soon after (reviewed in Cozzolino et al., 2012) and the link between the two, OS and MD, appeared most probable, if not obvious.

We will now briefly review recent evidence that OS and MD are found also in non-SOD1 linked ALS.

\section{OXIDATIVE STRESS IN ALS}

OS biomarkers have been repeatedly found in sporadic ALS patients, which may indicate that abnormal OS is relevant to the pathogenesis of this disease (D'Amico et al., 2013). A number of environmental factors may contribute to the generation of noxious free radicals, both Reactive Oxygen Species (ROS) and Reactive Nitrogen Species (RNS). For instance, transition metal-mediated OS has been proposed to contribute 
to ALS pathogenesis more than 10 years ago (reviewed in Carrí et al., 2003) and the role of metals is still discussed (Lovejoy and Guillemin, 2014). While no single pro-oxidant factor has emerged as crucially linked to sporadic ALS, recent genetic evidence reinforces the concept that OS may play a main role in familial ALS including in non-SOD1 ALS. For instance, expression of mutant valosin-containing protein (VCP, a member of the type II AAA+ ATPase family with a number of cellular functions including mitochondrial quality control, autophagy, vesicle transport and fusion, $26 \mathrm{~S}$ proteasome function, and assembly of peroxisomes) found in ALS patients makes human neuroblastoma SH-SY5Y cells susceptible to OS induced by treatment with L-buthionine sulfoximine, an inhibitor of the synthesis of glutathione (GSH; Hirano et al., 2014). GSH is a free-radical scavenger tripeptide which is among the main regulators of the intracellular redox state. Its levels are lower in the motor cortex of ALS patients than in healthy volunteers in vivo (Weiduschat et al., 2014) and decreasing GSH accelerates neurological deficit and mitochondrial pathology in the mutant SOD1 ALS mice model (Vargas et al., 2011). Furthermore, GSH depletion in cultured neurons induces the formation of cytoplasmic TDP-43 inclusions which are found in sporadic ALS patients (Iguchi et al., 2012).

Alterations in markers of OS other than GSH are found as a consequence of the expression of ALS-linked mutations. For instance, expression of mutant TDP-43 in a motor neuronlike cell line induces $\mathrm{OS}, \mathrm{MD}$ and nuclear accumulation of nuclear factor E2-related factor $2(\mathrm{Nrf} 2)$ that is an indicator and modulator of OS (Duan et al., 2010); in a yeast model, TDP43-expressing cells display increased markers of OS, apoptosis, and necrosis and these cytotoxic effects are potentiated upon expression of disease-associated variants (Braun et al., 2011) and Drosophila flies engineered for motoneuron-directed expression of TDP-43 have increased levels of protein carbonylation and Glutathione S transferase D1, both known markers of OS (Zhan et al., 2015). Overall these data indicate that OS is important also for TDP-43-triggered cell death, although the mechanism is still debated.

OS may not only increase the net production of ROS and RNS, but also affect protein conformation and structure, leading to the accumulation of the abnormal protein inclusions that are extensively described in ALS mouse models and patientderived tissue. Cysteine-mediated aggregation of mutant SOD1 has been widely studied and wild type SOD1 also hyperaggregates when oxidized (Guareschi et al., 2012). Treatments that deplete the cellular pool of GSH exacerbate mutSOD1s insolubility, whereas an overload of intracellular GSH or overexpression of glutaredoxins 1 and 2 significantly rescues mutSOD1s solubility in the cytosol and in mitochondria, respectively (Ferri et al., 2010). Interestingly, recent evidence suggests that also wild-type and mutant TDP-43 aggregation is caused by incorrect disulphide bonds involving Cys residues in one of its RNA recognition motifs, and that aggregation is promoted by OS (Cohen et al., 2012; Shodai et al., 2013).

Aggregation of TDP-43 (Parker et al., 2012) and FUS (Gerbino et al., 2013; Vance et al., 2013) proceeds, at least in part, through the stress granules (SGs) pathway. SGs are highly dynamic structures that are formed upon OS and contain RNA-binding proteins, transcription factors, RNA helicases and nucleases that work as sorting granules for mRNAs undergoing degradation, storage or translation (Baron et al., 2013 and references therein). SGs are found as a consequence of FUS mutation and mutated FUS is more rapidly directed to SGs after OS than wild type FUS (Bosco et al., 2010). Furthermore, TDP-43 is recruited to SGs in conditions of OS (Colombrita et al., 2009). These SGs may simply sequester a subset of mRNAs thus inducing cell dysfunction or serve as nucleation site for larger protein aggregates as the ones found in ALS patients.

Possibly representing a cellular response to this kind of aggregation and as a reaction against ER stress and unfolded protein response (UPR), protein disulfide isomerase (PDI) expression levels are upregulated in spinal cord tissue of ALS patients where PDI co-localizes with SOD1, TDP-43, and FUS (Atkin et al., 2008; Honjo et al., 2011; Farg et al., 2012; Walker et al., 2013). PDI co-localizes also with VAPB inclusions in a Drosophila melanogaster model of ALS (Tsuda et al., 2008). However, PDI itself contains an active site thiol group that is necessary for its activity and it is found oxidized through Snitrosylation in ALS (Walker et al., 2010), which may lead to a cycle of PDI upregulation and inactivation that perpetuates redox dysregulation and protein aggregation.

Interestingly, even mutant C9orf72 repeats may be related to OS. Indeed, similarly to what happens when expressing mutant SOD1, motor neurons differentiated from patients' iPSC (induced Pluripotent Stem Cells) and expressing expanded C9orf72 repeat display a significant induction of catalase, which is indicative of OS, and a significant change in levels of the mitochondrial transporter MTX3 (Kiskinis et al., 2014).

Mitochondria are a known target of OS because of their high dependance on membrane integrity and because they possess their own DNA and RNA that may be damaged by oxidation as well.

\section{MITOCHONDRIAL DAMAGE IN ALS}

Keeping in mind that mitochondria are not only a target of OS, but also the main site of production of ROS, it is obvious that OS may result from impairment of mitochondrial function.

A number of studies have reported altered mitochondrial respiratory complex activity in ALS tissues including postmortem brain and spinal cord tissue, patient lymphocytes, and in the SOD1 transgenic mouse model of ALS (for a review Cozzolino and Carrì, 2012; Tan et al., 2014). MD may follow the accumulation of aggregated SOD1 associated to mitochondria (Ferri et al., 2006) and result from the interaction of misfolded SOD1 with mitochondrial partners (Israelson et al., 2010; Li et al., 2010 and references therein). Evidence is accumulating that MD takes place also as a consequence of the expression of other ALS-related proteins, some of which are directly linked to the function of these organelles. For instance, mutations in VCP account for about $2 \%$ of familial ALS and $\mathrm{R} 155 \mathrm{H} /+$ VCP knock-in mice show signs of motor damage due to muscle denervation and degeneration accompanied by 
extensive accumulation of abnormal mitochondria in the intermyofibrillar space; in this model slow motor neuron loss is accompanied by TDP-43 accumulation and extensive aggregation of mitochondria (Yin et al., 2012; Nalbandian et al., 2013). In a 2013 study in VCP-deficient cells and in fibroblasts with VCP mutations, Bartolome et al. demonstrated that VCP deficiency causes significant mitochondrial uncoupling, leading to decreased mitochondrial membrane potential, increased mitochondrial oxygen consumption and reduction of cellular ATP production (Bartolome et al., 2013).

ALS may derive from mitochondrial DNA instability, as suggested in a study on a family with a late-onset phenotype including motor neuron disease and cognitive decline resembling frontotemporal dementia. Those patients carried a missense mutation in the CHCHD10 gene that encodes a mitochondrial protein located in the intermembrane space and enriched at cristae junctions. Muscle fibers from those patients are raggedred and cytochrome $c$ oxidase-negative with respiratory chain deficiency and abnormal assembly of complex V. Their fibroblasts have respiratory chain deficiency, mitochondrial ultrastructural alterations and fragmentation of the mitochondrial network (Bannwarth et al., 2014).

Neuronal mitochondrial abnormalities occur also in models of familial ALS associated with proteins that are (at least apparently) not related with these organelles. MD is observed together with oxidative damage and induction of mitophagy in the motor neuron-like NSC34 cell line expressing wild type or mutant TDP-43 (Duan et al., 2010; Hong et al., 2012). Mice expressing wild-type human TDP-43 show a pathology resembling ALS and motor neurons from these mice display cytoplasmic TDP-43 positive inclusions composed of mitochondria aggregates ( $\mathrm{Xu}$ et al., 2010), that may arise from defective intracellular trafficking and result in the reduction of mitochondria at nerve terminals of neuromuscular junctions (Shan et al., 2010). However, in mice expressing the mutant TDP43(A315T) protein, morphological abnormalities appear after the onset of transport defects (Magrané et al., 2014). In another study, MD in heterozygous TDP-43(A315TKi) animals is indicated by the formation of abnormal neuronal mitochondrial cristae and decrease in expression of Parkin and the fatty acid transporter CD36 along with an increase in fatty acids, HDL cholesterol, and glucose in the blood (Stribl et al., 2014). TDP-43 also perturbs ERmitochondria interactions and this is associated with disruption in cellular $\mathrm{Ca}^{2+}$ homeostasis (Stoica et al., 2014). Disturbance of mitochondrial $\mathrm{Ca}^{2+}$ transport in ALS have been widely described in SOD1 models in vitro and in vivo by the group of B. Keller (Jaiswal and Keller, 2009; Jaiswal et al., 2009) and by others; this aspect has been recently reviewed in detail (Barrett et al., 2014; Tadic et al., 2014) and thus we will not discuss it in this paper.

Not much evidence of functional MD in FUS-linked ALS is reported. However, mitochondria are disorganized in postmortem neurons from juvenile ALS patients (Huang et al., 2010) and shorter in cultured motor neurons expressing cytoplasmic FUS mutants (Tradewell et al., 2012). Furthermore, overexpression of ALS-linked human mutant FUS leads to Golgi fragmentation and mitochondria aggregation in rats (Huang et al., 2012).
How the overexpression or mutations of two proteins, TDP-43 and FUS, which are clearly involved in physiological mRNA metabolism, may result into MD in ALS is not clear at all.

One possibility is that misregulation of some step of RNA maturation leads to altered expression of proteins involved in mitochondria homeostasis. This may be the case for FUS, which transcriptionally regulates the expression of OS protection genes through the interaction with peroxisome proliferatoractivated receptor coactivator 1- $\alpha$ (PGC-1 $\alpha$; Sánchez-Ramos et al., 2011), that is a known regulator of mitochondrial biogenesis and function. In this context, MD may arise indirectly from modulation of Sirtuins (class III histone deacetylases). In fact PGC- $1 \alpha$ is downregulated in ALS patients and it is known that silencing of PGC1 $\alpha$ reduces the expression of SIRT3, which is the main mitochondrial deacetylase with a number of substrates (including SOD2), whose main function is to counteract ROS production and detoxification (Bause and Haigis, 2013), while overexpression of SIRT3 stimulates the expression of PGC1 $\alpha$ causing a further decrease of ROS in a positive feedback loop (Kong et al., 2010). Interestingly, both SIRT3 and PGC-1 $\alpha$ protect against mitochondrial fragmentation and neuronal cell death in rat spinal cord motor neurons overexpressing ALS-linked mutant SOD1-G93A (Song et al., 2013). Furthermore, nuclear SIRT1 also induces the expression of OS response genes and promotes mitochondrial biogenesis by activating PGC-1 $\alpha$ (Hall et al., 2013). Nonetheless, SIRT1 overexpression does not protect neurons against toxicity induced by mutant G93A-SOD1 (Valle et al., 2014 and reference therein).

Another possibility is that, similarly to mutant SOD1, TDP-43 and FUS have a pathological tendency to associate with mitochondria and to aggregate. Indeed, both wild type and a truncated form of TDP-43 were found localized in the mitochondria in NSC34 cells (Hong et al., 2012) and TDP-43 aggregates around mitochondria in a yeast model where altered mitochondrial respiratory activity is observed (Braun et al., 2011). Furthermore, FUS interacts with several proteins involved in mitochondrial metabolism and significantly reduces ATP levels in cells with FUS accumulation (Wang et al., 2015).

\section{CONCLUSIONS}

Previous failures in translating antioxidant and mitochondriaprotective strategies that were effective in the mutant SOD1 mouse model into positive clinical trials has cast a doubt on the real relevance of OS and MD in ALS and suggested that the SOD1 model represents only a fraction of a more heterogeneous population. However, new evidence has accumulated in recent years supporting the view that $\mathrm{MD}$ and $\mathrm{OS}$ play a role also in non-SOD1 ALS (Figure 1). This evidence is still somewhat sparse and often comes from studies in cell or invertebrate models that may represent only a few aspects of the human pathology. Nonetheless, while it has become clear that ALS is a complex disease with various presentation including an overlap with fronto-temporal dementia (Talbot, 2014), OS and $\mathrm{MD}$ may still represent a common denominator of motor neuron degeneration in ALS, and therefore a valuable target 


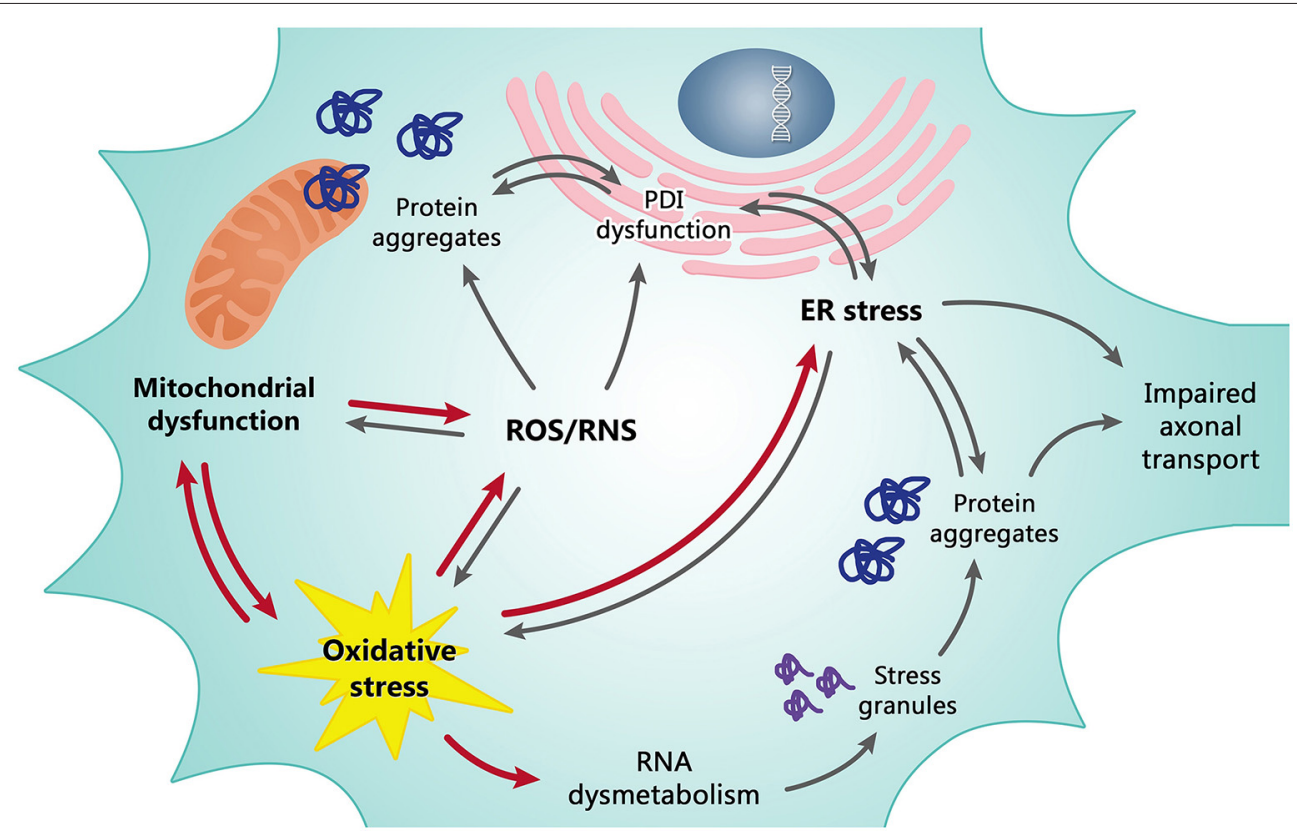

FIGURE 1 | Mitochondrial dysfunction and oxidative stress (OS) are tightly dependent on each other and are the basis of the redox dysregulation in ALS. Increased production of ROS/RNS, the ER stress and, at least in part, transcriptional dysregulation and abnormal RNA processing are all consequences of mitochondrial dysfunction and OS contributing to death of motor neurons. In turn, these pathological events cause other correlated detrimental effects as the formation of misfolded protein leading to insoluble cytosolic and mitochondrial aggregates, impaired axonal transport and alteration of the enzymatic activity of PDI. The line between cause and effect of individual events is however often difficult to draw since they are all tightly dependent/connected. Red arrows may be considered as primary causes, grey arrows as secondary causes/effects. that encourages transfer of new knowledge from research into preclinical testing.

\section{ACKNOWLEDGMENTS}

MTC is supported by ARiSLA (Project OligoALS) and by the Bruno and Ilse Frick Foundation for ALS research 2012 Award; MC is supported by Ministero della Salute (Project RF-20102309849) and by AriSLA (Project FUSMALS).

We wish to apologize to the many authors whose original contributions have not been cited owing to space restrictions.

\section{REFERENCES}

Atkin, J. D., Farg, M. A., Walker, A. K., McLean, C., Tomas, D., and Horne, M. K. (2008). Endoplasmic reticulum stress and induction of the unfolded protein response in human sporadic amyotrophic lateral sclerosis. Neurobiol. Dis. 30, 400-407. doi: 10.1016/j.nbd.2008.02.009

Bannwarth, S., Ait-El-Mkadem, S., Chaussenot, A., Genin, E. C., Lacas-Gervais, S., Fragaki, K., et al. (2014). A mitochondrial origin for frontotemporal dementia and amyotrophic lateral sclerosis through CHCHD10 involvement. Brain 137, 2329-2345. doi: 10.1093/brain/awu138

Baron, D. M., Kaushansky, L. J., Ward, C. L., Sama, R. R., Chian, R. J., Boggio, K. J., et al. (2013). Amyotrophic lateral sclerosis-linked FUS/TLS alters stress granule assembly and dynamics. Mol. Neurodegener. 8:30. doi: 10.1186/1750-1326-8-30

Barrett, E. F., Barrett, J. N., and David, G. (2014). Dysfunctional mitochondrial $\mathrm{Ca}(2+)$ handling in mutant SOD1 mouse models of fALS: integration of findings from motor neuron somata and motor terminals. Front. Cell. Neurosci. 8:184. doi: 10.3389/fncel.2014.00184

Bartolome, F., Wu, H. C., Burchell, V. S., Preza, E., Wray, S., Mahoney, C. J., et al. (2013). Pathogenic VCP mutations induce mitochondrial uncoupling and reduced ATP levels. Neuron 78, 57-64. doi: 10.1016/j.neuron.2013.02.028
Bause, A. S., and Haigis, M. C. (2013). SIRT3 regulation of mitochondrial oxidative stress. Exp. Gerontol. 48, 634-639. doi: 10.1016/j.exger.2012.08.007

Bosco, D. A., Lemay, N., Ko, H. K., Zhou, H., Burke, C., Kwiatkowski, T. J., et al. (2010). Mutant FUS proteins that cause amyotrophic lateral sclerosis incorporate into stress granules. Hum. Mol. Genet. 19, 4160-4175. doi: 10. 1093/hmg/ddq335

Braun, R. J., Sommer, C., Carmona-Gutierrez, D., Khoury, C. M., Ring, J., Büttner, S., et al. (2011). Neurotoxic 43-kDa TAR DNA-binding protein (TDP-43) triggers mitochondrion-dependent programmed cell death in yeast. J. Biol. Chem. 286, 19958-19972. doi: 10.1074/jbc.M110.194852

Carrí, M. T., Ferri, A., Cozzolino, M., Calabrese, L., and Rotilio, G. (2003). Neurodegeneration in amyotrophic lateral sclerosis: the role of oxidative stress and altered homeostasis of metals. Brain Res. Bull. 61, 365-374. doi: 10. 1016/s0361-9230(03)00179-5

Cohen, T. J., Hwang, A. W., Unger, T., Trojanowski, J. Q., and Lee, V. M. (2012). Redox signalling directly regulates TDP-43 via cysteine oxidation and disulphide cross-linking. EMBO J. 31, 1241-1252. doi: 10.1038/emboj.2011.471

Colombrita, C., Zennaro, E., Fallini, C., Weber, M., Sommacal, A., Buratti, E., et al. (2009). TDP-43 is recruited to stress granules in conditions of oxidative insult. J. Neurochem. 111, 1051-1061. doi: 10.1111/j.1471-4159.2009.06383.x

Cozzolino, M., and Carrì, M. T. (2012). Mitochondrial dysfunction in ALS. Prog. Neurobiol. 97, 54-66. doi: 10.1016/j.pneurobio.2011.06.003

Cozzolino, M., Pesaresi, M. G., Gerbino, V., Grosskreutz, J., and Carrì, M. T. (2012). Amyotrophic lateral sclerosis: new insights into underlying molecular mechanisms and opportunities for therapeutic intervention. Antioxid. Redox Signal. 17, 1277-1330. doi: 10.1089/ars.2011.4328

D’Amico, E., Factor-Litvak, P., Santella, R. M., and Mitsumoto, H. (2013). Clinical perspective on oxidative stress in sporadic amyotrophic lateral sclerosis. Free Radic. Biol. Med. 65, 509-527. doi: 10.1016/j.freeradbiomed.2013. 06.029

Duan, W., Li, X., Shi, J., Guo, Y., Li, Z., and Li, C. (2010). Mutant TAR DNA-binding protein-43 induces oxidative injury in motor neuron-like cell. Neuroscience 169, 1621-1629. doi: 10.1016/j.neuroscience.2010.06.018 
Farg, M. A., Soo, K. Y., Walker, A. K., Pham, H., Orian, J., Horne, M. K., et al. (2012). Mutant FUS induces endoplasmic reticulum stress in amyotrophic lateral sclerosis and interacts with protein disulfideisomerase. Neurobiol. Aging 33, 2855-2868. doi: 10.1016/j.neurobiolaging.2012. 02.009

Ferri, A., Cozzolino, M., Crosio, C., Nencini, M., Casciati, A., Gralla, E. B., et al. (2006). Familial ALS-superoxide dismutases associate with mitochondria and shift their redox potentials. Proc. Natl. Acad. Sci. U S A 103, 13860-13865. doi: 10.1073/pnas.0605814103

Ferri, A., Fiorenzo, P., Nencini, M., Cozzolino, M., Pesaresi, M. G., Valle, C., et al. (2010). Glutaredoxin 2 prevents aggregation of mutant SOD1 in mitochondria and abolishes its toxicity. Hum. Mol. Genet. 19, 4529-4542. doi: $10.1093 / \mathrm{hmg} / \mathrm{ddq} 383$

Gerbino, V., Carrì, M. T., Cozzolino, M., and Achsel, T. (2013). Mislocalised FUS mutants stall spliceosomal snRNPs in the cytoplasm. Neurobiol. Dis. 55, 120128. doi: 10.1016/j.nbd.2013.03.003

Guareschi, S., Cova, E., Cereda, C., Ceroni, M., Donetti, E., Bosco, D. A., et al. (2012). An over-oxidized form of superoxide dismutase found in sporadic amyotrophic lateral sclerosis with bulbar onset shares a toxic mechanism with mutant SOD1. Proc. Natl. Acad. Sci. U S A 109, 5074-5079. doi: 10.1073/pnas. 1115402109

Hall, J. A., Dominy, J. E., Lee, Y., and Puigserver, P. (2013). The sirtuin family's role in aging and age-associated pathologies. J. Clin. Invest. 123, 973-979. doi: 10. 1172/JCI64094

Hirano, M., Nakamura, Y., Saigoh, K., Sakamoto, H., Ueno, S., Isono, C., et al. (2014). VCP gene analyses in Japanese patients with sporadic amyotrophic lateral sclerosis identify a new mutation. Neurobiol. Aging doi: 10.1016/j. neurobiolaging.2014.10.012. [Epub ahead of print].

Hong, K., Li, Y., Duan, W., Guo, Y., Jiang, H., Li, W., et al. (2012). Full-length TDP43 and its C-terminal fragments activate mitophagy in NSC34 cell line. Neurosci. Lett. 530, 144-149. doi: 10.1016/j.neulet.2012.10.003

Honjo, Y., Kaneko, S., Ito, H., Horibe, T., Nagashima, M., Nakamura, M., et al. (2011). Protein disulfide isomerase-immunopositive inclusions in patients with amyotrophic lateral sclerosis. Amyotroph. Lateral. Scler. 12, 444-450. doi: 10. 3109/17482968.2011.594055

Huang, C., Tong, J., Bi, F., Wu, Q., Huang, B., Zhou, H., et al. (2012). Entorhinal cortical neurons are the primary targets of FUS mislocalization and ubiquitin aggregation in FUS transgenic rats. Hum. Mol. Genet. 21, 4602-4614. doi: 10. 1093/hmg/dds299

Huang, E. J., Zhang, J., Geser, F., Trojanowski, J. Q., Strober, J. B., Dickson, D. W., et al. (2010). Extensive FUS-immunoreactive pathology in juvenile amyotrophic lateral sclerosis with basophilic inclusions. Brain Pathol. 20, 1069-1076. doi: 10. 1111/j.1750-3639.2010.00413.x

Iguchi, Y., Katsuno, M., Takagi, S., Ishigaki, S., Niwa, J., Hasegawa, M., et al. (2012). Oxidative stress induced by glutathione depletion reproduces pathological modifications of TDP-43 linked to TDP-43 proteinopathies. Neurobiol. Dis. 45, 862-870. doi: 10.1016/j.nbd.2011.12.002

Israelson, A., Arbel, N., Da Cruz, S., Ilieva, H., Yamanaka, K., Shoshan-Barmatz, V., et al. (2010). Misfolded mutant SOD1 directly inhibits VDAC1 conductance in a mouse model of inherited ALS. Neuron 67, 575-587. doi: 10.1016/j.neuron. 2010.07.019

Jaiswal, M. K., and Keller, B. U. (2009). Cu/Zn superoxide dismutase typical for familial amyotrophic lateral sclerosis increases the vulnerability of mitochondria and perturbs Ca2+ homeostasis in SOD1G93A mice. Mol. Pharmacol. 75, 478489. doi: 10.1124/mol.108.050831

Jaiswal, M. K., Zech, W. D., Goos, M., Leutbecher, C., Ferri, A., Zippelius, A., et al. (2009). Impairment of mitochondrial calcium handling in a mtSOD1 cell culture model of motoneuron disease. BMC Neurosci. 10:64. doi: 10.1186/14712202-10-64

Kiskinis, E., Sandoe, J., Williams, L. A., Boulting, G. L., Moccia, R., Wainger, B. J., et al. (2014). Pathways disrupted in human ALS motor neurons identified through genetic correction of mutant SOD1. Cell Stem Cell 14, 781-795. doi: 10. 1016/j.stem.2014.03.004

Kong, X., Wang, R., Xue, Y., Liu, X., Zhang, H., Chen, Y., et al. (2010). Sirtuin 3, a new target of PGC-1alpha, plays an important role in the suppression of ROS and mitochondrial biogenesis. PLoS One 5:e11707. doi: 10.1371/journal.pone. 0011707

Li, Q., Vande Velde, C., Israelson, A., Xie, J., Bailey, A. O., Dong, M. Q., et al. (2010). ALS-linked mutant superoxide dismutase 1 (SOD1) alters mitochondrial protein composition and decreases protein import. Proc. Natl. Acad. Sci. U S A 107, 21146-21151. doi: 10.1073/pnas.1014862107

Lovejoy, D. B., and Guillemin, G. J. (2014). The potential for transition metalmediated neurodegeneration in amyotrophic lateral sclerosis. Front. Aging Neurosci. 6:173. doi: 10.3389/fnagi.2014.00173

Magrané, J., Cortez, C., Gan, W. B., and Manfredi, G. (2014). Abnormal mitochondrial transport and morphology are common pathological denominators in SOD1 and TDP43 ALS mouse models. Hum. Mol. Genet. 23, 1413-1424. doi: 10.1093/hmg/ddt528

Marangi, G., and Traynor, B. J. (2014). Genetic causes of amyotrophic lateral sclerosis: new genetic analysis methodologies entailing new opportunities and challenges. Brain Res. doi: 10.1016/j.brainres.2014.10.009. [Epub ahead of print].

Nalbandian, A., Llewellyn, K. J., Badadani, M., Yin, H. Z., Nguyen, C., Katheria, V., et al. (2013). A progressive translational mouse model of human valosincontaining protein disease: the VCP(R155H/+) mouse. Muscle Nerve 47, 260 270. doi: $10.1002 /$ mus. 23522

Parker, S. J., Meyerowitz, J., James, J. L., Liddell, J. R., Crouch, P. J., Kanninen, K. M., et al. (2012). Endogenous TDP-43 localized to stress granules can subsequently form protein aggregates. Neurochem. Int. 60, 415-424. doi: 10.1016/j.neuint. 2012.01.019

Sánchez-Ramos, C., Tierrez, A., Fabregat-Andrés, O., Wild, B., Sánchez-Cabo, F., Arduini, A., et al. (2011). PGC-1 $\alpha$ regulates translocated in liposarcoma activity: role in oxidative stress gene expression. Antioxid. Redox Signal. 15, 325-337. doi: 10.1089/ars.2010.3643

Shan, X., Chiang, P. M., Price, D. L., and Wong, P. C. (2010). Altered distributions of Gemini of coiled bodies and mitochondria in motor neurons of TDP43 transgenic mice. Proc. Natl. Acad. Sci. U S A 107, 16325-16330. doi: 10. 1073/pnas. 1003459107

Shodai, A., Morimura, T., Ido, A., Uchida, T., Ayaki, T., Takahashi, R., et al. (2013). Aberrant assembly of RNA recognition motif 1 links to pathogenic conversion of TAR DNA-binding protein of 43 kDa (TDP-43). J. Biol. Chem. 288, 1488614905. doi: 10.1074/jbc.M113.451849

Song, W., Song, Y., Kincaid, B., Bossy, B., and Bossy-Wetzel, E. (2013). Mutant SOD1G93A triggers mitochondrial fragmentation in spinal cord motor neurons: neuroprotection by SIRT3 and PGC-1 $\alpha$. Neurobiol. Dis. $51,72-81$. doi: 10.1016/j.nbd.2012.07.004

Stoica, R., De Vos, K. J., Paillusson, S., Mueller, S., Sancho, R. M., Lau, K. F., et al. (2014). ER-mitochondria associations are regulated by the VAPB-PTPIP51 interaction and are disrupted by ALS/FTD-associated TDP-43. Nat. Commun. 5:3996. doi: 10.1038/ncomms4996

Stribl, C., Samara, A., Trüembach, D., Peis, R., Neumann, M., Fuchs, H., et al. (2014). Mitochondrial dysfunction and decrease in body weight of a transgenic knock-in mouse model for TDP-43. J. Biol. Chem. 289, 10769-10784. doi: 10. 1074/jbc.M113.515940

Tadic, V., Prell, T., Lautenschlaeger, J., and Grosskreutz, J. (2014). The ER mitochondria calcium cycle and ER stress response as therapeutic targets in amyotrophic lateral sclerosis. Front. Cell. Neurosci. 8:147. doi: 10.3389/fncel. 2014.00147

Talbot, K. (2014). Amyotrophic lateral sclerosis: cell vulnerability or system vulnerability? J. Anat. 224, 45-51. doi: 10.1111/joa.12107

Tan, W., Pasinelli, P., and Trotti, D. (2014). Role of mitochondria in mutant SOD1 linked amyotrophic lateral sclerosis. Biochim. Biophys. Acta 1842, 1295-1301. doi: 10.1016/j.bbadis.2014.02.009

Tradewell, M. L., Yu, Z., Tibshirani, M., Boulanger, M. C., Durham, H. D., and Richard, S. (2012). Arginine methylation by PRMT1 regulates nuclearcytoplasmic localization and toxicity of FUS/TLS harbouring ALS-linked mutations. Hum. Mol. Genet. 21, 136-149. doi: 10.1093/hmg/ddr448

Tsuda, H., Han, S. M., Yang, Y., Tong, C., Lin, Y. Q., Mohan, K., et al. (2008). The amyotrophic lateral sclerosis 8 protein VAPB is cleaved, secreted and acts as a ligand for Eph receptors. Cell 133, 963-977. doi: 10.1016/j.cell.2008.04.039

Valle, C., Salvatori, I., Gerbino, V., Rossi, S., Palamiuc, L., René, F., et al. (2014). Tissue-specific deregulation of selected HDACs characterizes ALS progression in mouse models: pharmacological characterization of SIRT1 and SIRT2 pathways. Cell Death Dis. 5:e1296. doi: 10.1038/cddis.2014.247

Vance, C., Scotter, E. L., Nishimura, A. L., Troakes, C., Mitchell, J. C., Kathe, C., et al. (2013). ALS mutant FUS disrupts nuclear localization and sequesters wildtype FUS within cytoplasmic stress granules. Hum. Mol. Genet. 22, 2676-2688. doi: $10.1093 / \mathrm{hmg} / \mathrm{ddt} 117$ 
Vargas, M. R., Johnson, D. A., and Johnson, J. A. (2011). Decreased glutathione accelerates neurological deficit and mitochondrial pathology in familial ALSlinked hSOD1(G93A) mice model. Neurobiol. Dis. 43, 543-551. doi: 10.1016/j. nbd.2011.04.025

Walker, A. K., Farg, M. A., Bye, C. R., McLean, C. A., Horne, M. K., and Atkin, J. D. (2010). Protein disulphide isomerase protects against protein aggregation and is S-nitrosylated in amyotrophic lateral sclerosis. Brain 133, 105-116. doi: 10. 1093/brain/awp267

Walker, A. K., Soo, K. Y., Sundaramoorthy, V., Parakh, S., Ma, Y., Farg, M. A., et al. (2013). ALS-associated TDP-43 induces endoplasmic reticulum stress, which drives cytoplasmic TDP-43 accumulation and stress granule formation. PLoS One 8:e81170. doi: 10.1371/journal.pone.0081170

Wang, T., Jiang, X., Chen, G., and Xu, J. (2015). Interaction of amyotrophic lateral sclerosis/frontotemporal lobar degeneration-associated fused-in-sarcoma with proteins involved in metabolic and protein degradation pathways. Neurobiol. Aging 36, 527-535. doi: 10.1016/j.neurobiolaging.2014.07.044

Weiduschat, N., Mao, X., Hupf, J., Armstrong, N., Kang, G., Lange, D. J., et al. (2014). Motor cortex glutathione deficit in ALS measured in vivo with the J-editing technique. Neurosci. Lett. 570, 102-107. doi: 10.1016/j.neulet.2014. 04.020

Xu, Y. F., Gendron, T. F., Zhang, Y. J., Lin, W. L., D’Alton, S., Sheng, H., et al. (2010). Wild-type human TDP-43 expression causes TDP-43 phosphorylation, mitochondrial aggregation, motor deficits and early mortality in transgenic mice. J. Neurosci. 30, 10851-10859. doi: 10.1523/JNEUROSCI.1630-10. 2010
Yin, H. Z., Nalbandian, A., Hsu, C. I., Li, S., Llewellyn, K. J., Mozaffar, T., et al. (2012). Slow development of ALS-like spinal cord pathology in mutant valosin-containing protein gene knock-in mice. Cell Death Dis. 3:e374. doi: 10. 1038/cddis.2012.115

Zhan, L., Xie, Q., and Tibbetts, R. S. (2015). Opposing roles of p38 and JNK in a Drosophila model of TDP-43 proteinopathy reveal oxidative stress and innate immunity as pathogenic components of neurodegeneration. Hum. Mol. Genet. 24, 757-772. doi: $10.1093 / \mathrm{hmg} / \mathrm{ddu} 493$

Conflict of Interest Statement: The authors declare that the research was conducted in the absence of any commercial or financial relationships that could be construed as a potential conflict of interest.

Received: 23 December 2014; accepted: 27 January 2015; published online: 17 February 2015.

Citation: Carrì MT, Valle C, Bozzo F and Cozzolino M (2015) Oxidative stress and mitochondrial damage: importance in non-SOD1 ALS. Front. Cell. Neurosci. 9:41. doi: 10.3389/fncel.2015.00041

This article was submitted to the journal Frontiers in Cellular Neuroscience.

Copyright (c) 2015 Carrì, Valle, Bozzo and Cozzolino. This is an open-access article distributed under the terms of the Creative Commons Attribution License (CC BY). The use, distribution and reproduction in other forums is permitted, provided the original author(s) or licensor are credited and that the original publication in this journal is cited, in accordance with accepted academic practice. No use, distribution or reproduction is permitted which does not comply with these terms. 